\title{
Levodopa improves handwriting and instrumental tasks in previously treated patients with Parkinson's disease
}

\author{
Thomas Müller ${ }^{1}$ [ $\cdot$ Ali Harati $^{2}$
}

Received: 18 May 2020 / Accepted: 13 August 2020 / Published online: 19 August 2020

(c) The Author(s) 2020

\begin{abstract}
Motor symptoms in patients with Parkinson's disease may be determined with instrumental tests and rating procedures. Their outcomes reflect the functioning and the impairment of the individual patient when patients are tested off and on dopamine substituting drugs. Objectives were to investigate whether the execution speed of a handwriting task, instrumentally assessed fine motor behavior, and rating scores improve after soluble levodopa application. 38 right-handed patients were taken off their regular drug therapy for at least $12 \mathrm{~h}$ before scoring, handwriting, and performance of instrumental devices before and $1 \mathrm{~h}$ after $100 \mathrm{mg}$ levodopa intake. The outcomes of all performed procedures improved. The easy-to-perform handwriting task and the instrumental tests demand for fast and precise execution of movement sequences with considerable cognitive load in the domains' attention and concentration. These investigations may serve as additional tools for the testing of the dopaminergic response.
\end{abstract}

Keywords Levodopa $\cdot$ Parkinson's disease $\cdot$ Motor behavior $\cdot$ Handwriting

\section{Introduction}

Neurological examination of patients with Parkinson's disease (PD) includes several procedures that determine the impaired function of motor behavior. One is the performance of clinical scoring of motor symptoms with validated rating scales, like the Unified Parkinson's disease Rating Scale (UPDRS) (Fahn et al. 1987). However, this evaluation of motor impairment in PD may vary between different examiners and may relatively be insensitive to subtle modifications. The subjective impression of the patient by the rating neurologist may additionally impact outcomes (Goetz et al. 2008). Accordingly, quantitative standardized instrumental procedures for objective assessment of motor behavior have been developed over the years (Lee et al. 2016; Lopane et al.

Thomas Müller

th.mueller@alexianer.de;

thomas.mueller@ruhr-uni-bochum.de

1 Department of Neurology, St. Joseph Hospital BerlinWeissensee, Gartenstr. 1, 13088 Berlin, Germany

2 Private Practice for Neurosurgery, MVZ PAN Institute $\mathrm{GmbH}$ within the Department of Neurosurgery, Pan Klinik Am Neumarkt, Zeppelinstr 1 Neumarkt-Galerie, 50667 Cologne, Germany
2018). The employed technical methods aim to assess motor symptoms, particularly tremor and slowness of movement, which is considerably biased by rigidity (Goetz et al. 2008; van Uem et al. 2016; Li et al. 2018; Lopane et al. 2018; Bertoli et al. 2019). Particularly tremor reduces the precision of aimed movements, whereas bradykinesia and rigidity support delay or failure to initiate a willed movement and slowness of an ongoing motion sequence (Lalonde and Botez-Marquard 1997; Haaland et al. 2004). This triad of main cardinal motor symptoms in PD patients influences instrumentally assessed execution of complex movement series (Müller et al. 2017). They are sensitive to dopamine substitution in PD patients, as it has been shown with the peg insertion paradigm, which resembles the rather popular, simpler purdue pegboard task (Müller et al. 2005). As an example, instrumental assessment of conduction of complex movement sequences showed good test-retest reliability and correlated to the nigrostriatal dopamine deficit in PD (Pal et al. 2001; Müller et al. 2002). The outcomes of employed instrumental methods reflected altered motor behavior not only in PD, but also in multiple sclerosis or Huntington's disease (as examples: (Ringendahl 2002; Andrich et al. 2007)). Execution of more complex movement series involves higher cortical functions and dopamine sensitive mesolimbic structures. The necessary high cognitive 
load also demands attention and motivation (Nieoullon and Coquerel 2003; Bidet-Ildei et al. 2011; Trujillo et al. 2019). Similar cognitive efforts with a hypothetical involvement of resembling brain structures are necessary when one writes a sentence (Gangadhar et al. 2009). It is well known that not only micrographia, which is abnormal small, cramped handwriting or the progression to progressively smaller handwriting, but also bradykinesia and rigidity influence the handwriting procedure in PD patients (Lalonde et al. 1997; Nieoullon et al. 2003; Lange et al. 2006; Broeder et al. 2014; Nackaerts et al. 2017b). To a certain extent, a resembling instrumental task is the performance of a line tracing task, which asks the individual to follow a given path (Fig. 1). Measurements concern the execution velocity, the number, and duration of contacts to the path (Müller et al. 2005). The execution of instrumental tests in combination with a rating procedure may provide additional useful information on the functioning and the impairment of the individual PD patient in particular when patients are tested off and on medication to evaluate the dopaminergic response (Gelb et al. 1999). The objective was to determine whether outcomes of aforementioned instrumental tests and a standardized writing task may reflect the dopaminergic response similar to the improvement of clinical rating scale scores following levodopa/benserazide application (Müller et al. 2002, 2003, 2017).

\section{Methods}

\section{Subjects}

Participants were 38 treated, right-handed, idiopathic PD patients (see Table 1) without unpredictable motor fluctuations. Individuals with medical conditions, which may affect the outcomes of the performed instrumental tests, were excluded.
Table 1 Characteristics of participating PD patients

\begin{tabular}{ll}
\hline Age & $61.15 \pm 18.54$ years \\
Sex & 20 men; 18 women \\
Duration of PD & $4.26 \pm 4.86$ years \\
Age of onset & $53.87 \pm 13.01$ years \\
LEED & $386.45 \pm 261.70 \mathrm{mg}$ \\
HYS & $2.01 \pm 0.85$ \\
UPDRS I & $2.63 \pm 2.06$ \\
UPDRS II & $9.84 \pm 6.34$ \\
UPDRS IV & $1.09 \pm 0.12$ \\
UPDRS III right & $10.37 \pm 5.65$ \\
UPDRS III left & $9.63 \pm 5.06$ \\
PD dominance & 8 equal, 14 left, 16 right \\
\hline
\end{tabular}

HYS Hoehn and Yahr Scale, LEED L-dopa equivalent daily dose, $P D$ dominance most affected side by the disease process (equal $=$ equal on both sides, left $=$ left sided, right $=$ right sided), UPDRS I unified Parkinson's disease rating scale mental behavior, UPDRS II unified Parkinson's disease rating scale activities of daily living, UPDRS III unified Parkinson's disease rating scale motor examination, UPDRS IV unified Parkinson's disease rating scale motor complications, UPDRS III right/left items 20, 21, 22, 23, 24, 25, 26 (right/left) of unified Parkinson's disease rating scale

\section{Design}

Hospitalized patients were taken off their regular PD drug therapy for at least $12 \mathrm{~h}$ before the rating and execution of devices. First the rating (A.H.), second the handwriting test (A.H.), and third the assessments with the devices [standardized sequence: peg insertion [first], line tracing [second], were performed (technicians)]. Then, the patients received one tablet of $100 \mathrm{mg}$ levodopa (L-dopa)/25 mg benserazide, dissolved in $100 \mathrm{ml}$ water (Madopar $\mathrm{LT}^{\circledR}$ ). One hour later, UPDRS III rating and the instrumental tests in the same sequence were again executed. To minimize learning and training effects, all PD patients were allowed to practice for $1 \mathrm{~min}$ on the day before with all instrumental tests.

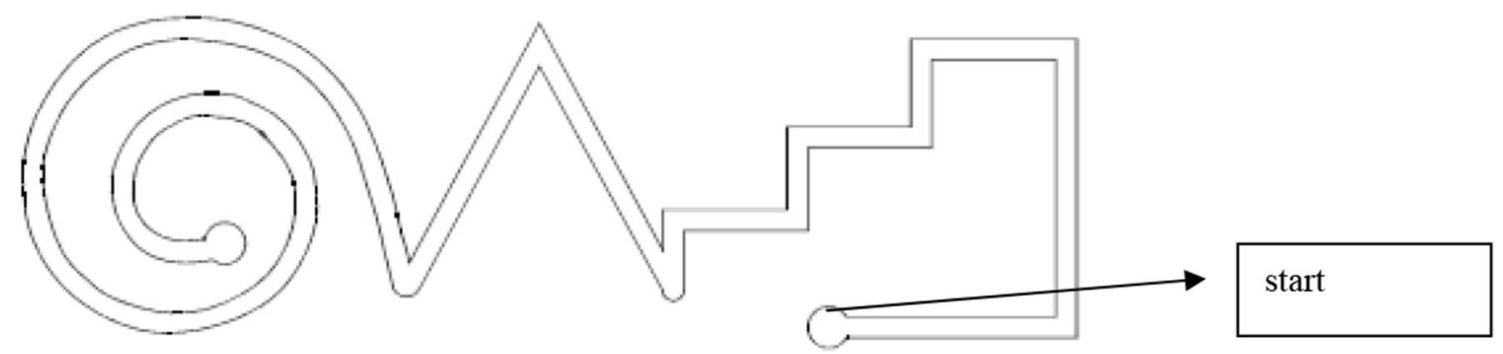

Fig. 1 Graph from the line tracing task ( modified from https://psydok.psycharchives.de/jspui/bitstream/20.500.11780/1018/1/Mls.pdf, page 7) 


\section{Rating}

Motor symptoms were scored with the part motor examination (III) of the UPDRS (Fahn et al. 1987).

\section{Writing test}

The participants were asked to sit down in a comfortable position and then to write the same sentence, consisting of 5 words with 24 characters ("Bochum ist eine schöne Stadt"), for three times on white paper without lines. Initially, subjects were asked to write this sentence two times. The patients were instructed to write this sentence with a comfortably speed like in the daily routine and not write this sentence as fast as possible. Then, the assessment of the needed interval for the handwriting task was performed. A stopwatch with $100 \mathrm{~ms}$ accuracy was employed.

\section{Instrumental tests}

\section{Peg insertion}

Subjects were instructed to transfer 25 pegs (diameter $2.5 \mathrm{~mm}$, length $5 \mathrm{~cm}$ ) from a rack into one of 25 holes (diameter $2.8 \mathrm{~mm}$ ) in a computer-based contact board individually and as quickly as possible. The distance between rack and appropriate holes was exactly $32 \mathrm{~cm}$. The board was positioned in the middle and the task was carried out with the right hand only. When transferring each peg from rack to hole, elbows were allowed to be in contact with the table. The interval between the inserting of the first and the last pin was measured by a computer with an accuracy of $100 \mathrm{~ms}$ (Müller et al. 2005). In case of dropping of one pin, the instruction was to repeat this task.

\section{Line tracing}

The patient was asked to follow a grooved path with a stylus as exact and fast as possible from the right to the left side with the right hand one time only. The total test duration, the number of contacts, and the length of contacts to the panel interfacing with a computer, which recorded all these parameters, were assessed (Müller et al. 2005). Intervals were determined with $100 \mathrm{~ms}$ accuracy.

\section{Statistics}

A non-parametric data distribution was shown according to the Kolmogorov-Smirnov test outcomes mainly. Therefore, non-parametric tests were only employed for this exploratory analysis. The Wilcoxon matched-pairs test was used for comparisons and Spearman rank correlation for correlation analysis. Suitable items of UPDRS part III were selected for calculation of subscores, i.e., the UPDRS III score of the right arm (items: 20, 21, 22, 23, 24, 25 [right arm]) only. The differences between the outcomes of the two assessment moments were calculated according to the formula: outcome before L-dopa/benserazide intake-outcome $1 \mathrm{~h}$ after L-dopa/benserazide = difference. Four different assessment tools, the peg insertion task, the line tracing paradigm, the writing test, and the UPDRS III rating were performed. Therefore, the significance level was corrected to $\mathrm{p}<0.0125$ for both comparisons and the correlation analysis. P-values between 0.0125 and 0.05 were discussed as a significant trend.

\section{Ethics}

The study was approved by the local institutional ethics committee of the Ruhr University of Bochum. The study was performed in the Department of Neurology, St Joseph Hospital (Head at that time: Professor Dr. H. Przuntek). The investigation represented a non-interventional study, i.e., the rules imposed for this observational plan did not interfere with the physician's common therapy. Patient's written informed consent regarding the forwarding and storing of medical data according to GDPR laws was obtained.

\section{Results}

\section{Comparisons}

As to be expected, there was a decrease and thus an amelioration of the UPDRS part III total score (before L-dopa/ benserazide intake [before]: $27.92 \pm 13.90$ [mean $\pm \mathrm{SD}$ ]; 1 $\mathrm{h}$ after L-dopa intake [after]: 18.95 $\pm 12.37, p=<0.0001)$. Accordingly, the UPDRS part III right arm subscore also went down and improved (Fig. 2a, $p=<0.0001$ ). Table 2 shows the outcomes of further UPDRS III subscores. The interval for the writing task performance declined (Table 2, Fig. 2b). The execution of the instrumental tests also improved after L-dopa/benserazide intake (Table 2; peg insertion outcome right side: Fig. 2c; line tracing errors: Fig. 2d; line tracing duration of errors: Fig. 2e; line tracing total interval: Fig. 2f).

\section{Correlations analysis}

The results are shown in Table 3. Only significant outcomes and associations with a significant trend are reported.

\section{Peg insertion}

The outcomes were associated with the UPDRS III right arm score, the writing task, and the line tracing duration of errors 
Fig. 2 Decline of the UPDRS part III arm scores (a), writing interval (b), peg insertion result (c), number of line tracing errors (d), line tracing duration of errors (e), interval of line tracing (f) before and $1 \mathrm{~h}$ after intake of 100-mg L-dopa/25-mg benserazide. UPDRS III motor examination (III) of the Unified Parkinson's disease rating scale, $y$-axis of $\mathbf{b}, \mathbf{c}, \mathbf{e}, \mathbf{f}$ describes data with 100-ms accuracy
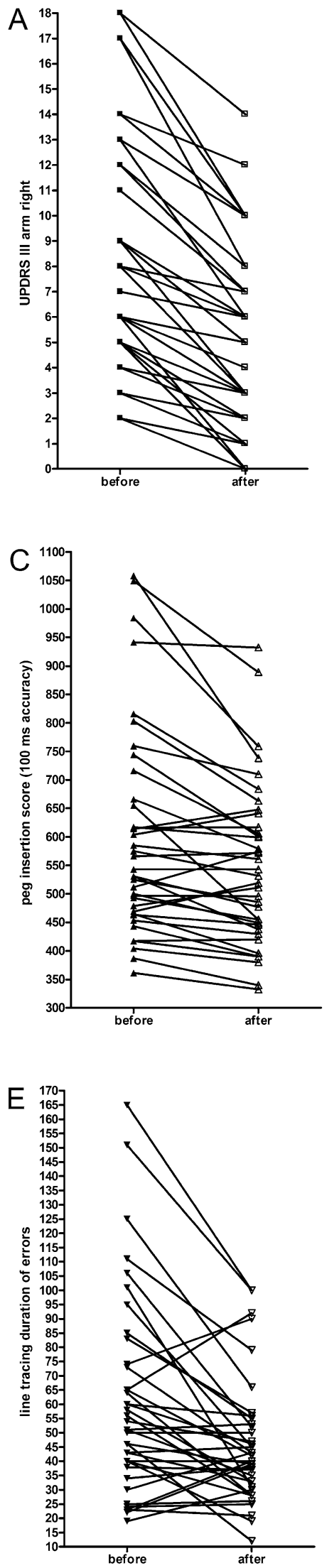
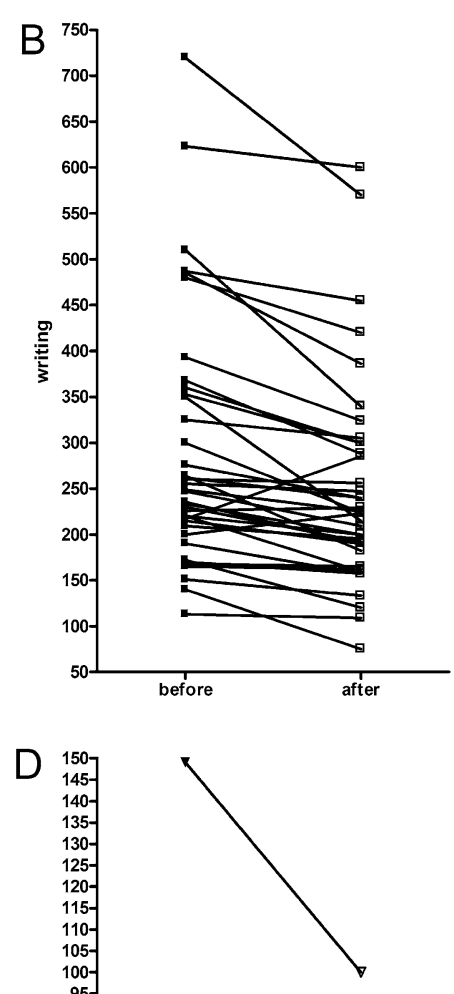

.
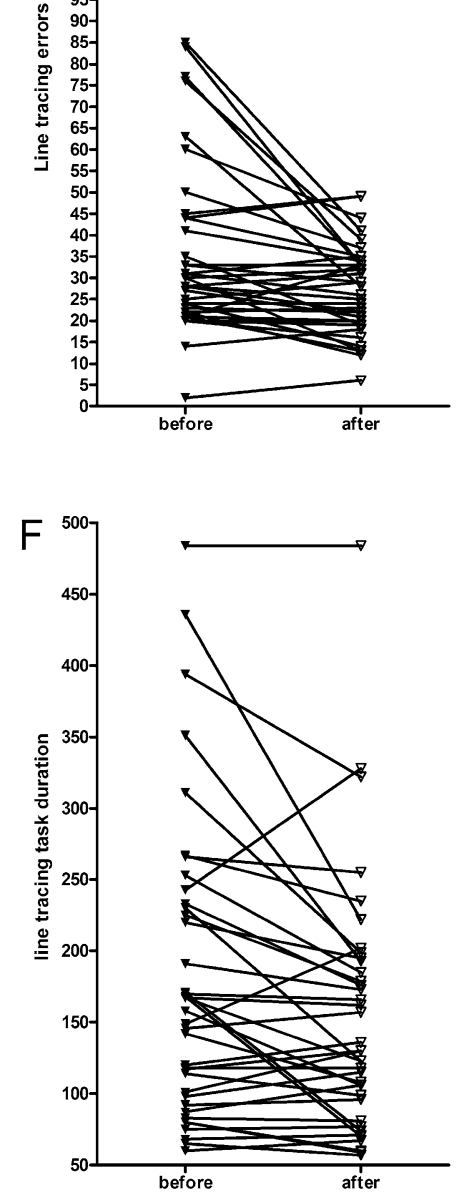
Table 2 Comparisons of data before and after L-dopa intake

\begin{tabular}{lcccc}
\hline & \multicolumn{1}{l}{ Before } & After & Difference & $p$ \\
\hline UPDRS III & $27.42 \pm 13.90$ & $18.95 \pm 12.37$ & $8.97 \pm 6.64$ & $<0.0001$ \\
UPDRS III right arm & $8.24 \pm 4.62$ & $5 \pm 3.57$ & $3.24 \pm 2.13$ & $<0.0001$ \\
UPDRS III right arm rigidity & $1.40 \pm 0.89$ & $0.95 \pm 0.90$ & $0.45 \pm 0.64$ & 0.0005 \\
UPDRS III right arm bradykinesia & $4.63 \pm 2.78$ & $2.95 \pm 2.44$ & $1.68 \pm 1.70$ & $<0.0001$ \\
UPDRS III right arm tremor & $1.95 \pm 1.87$ & $1.03 \pm 1.26$ & $0.92 \pm 1.04$ & $<0.0001$ \\
Writing & $290.55 \pm 134.39$ & $249.16 \pm 113.42$ & $41.39 \pm 44.99$ & $<0.0001$ \\
Peg insertion & $596.74 \pm 181.16$ & $543.37 \pm 140.13$ & $53.37 \pm 78.37$ & 0.0002 \\
Line tracing errors & $38.24 \pm 26.64$ & $28.95 \pm 15.42$ & $9.29 \pm 16.19$ & 0.0015 \\
Line tracing duration of errors & $61.55 \pm 34.63$ & $45.87 \pm 21.39$ & $15.68 \pm 24.22$ & 0.0011 \\
Line tracing total interval & $181.58 \pm 104.35$ & $153.66 \pm 86.56$ & $27.92 \pm 56.32$ & 0.0055 \\
\hline
\end{tabular}

All data are given as mean \pm standard deviation

After $1 \mathrm{~h}$ after intake of $100 \mathrm{mg} \mathrm{L-dopa} / 25 \mathrm{mg}$ benserazide, before before intake of 100-mg L-dopa/25-mg benserazide, $p p$ value; data of instrumental tests and writing are given with 100-ms accuracy except line tracing number of errors; UPDRS III right arm, items 20, 21, 22, 23, 24, 25 (right arm) of Unified Parkinson's Disease Rating Scale; UPDRS III right arm rigidity; item 22 (right arm) of Unified Parkinson's Disease Rating Scale; UPDRS III right arm bradykinesia; items 23, 24, 25 (right arm) of Unified Parkinson's Disease Rating Scale; UPDRS III right arm tremor; items 20, 21 (right arm) of Unified Parkinson's Disease Rating Scale before L-dopa intake (Table 3; lines 1, 2, 7, 12). These associations were also found following L-dopa intake with the exception of the correlation between the writing outcomes and peg insertion (Table 3; lines 17, 19, 21).

\section{Line tracing}

There were close relationships between each of the determined parameters of the line tracing task before L-dopa intake (Table 3; lines 13-15). The number of these significant relations between all parameters of the line tracing task went down after L-dopa intake (Table 3; line 20). Nearly all the computed differences of each line tracing parameter correlated with each other, line tracing errors, and line tracing duration of errors showed a significant trend only (Table 3; lines 24-26). There was a significant trend for a correlation between the line tracing duration of error results and the writing results after L-dopa intake only (Table 3, line 16).

\section{UPDRS part III right arm score and further UPDRS III subscores}

The rating scores correlated with the peg insertion results and with the line tracing duration of errors at baseline (Table 3; lines 2, 4, 8), and bradykinesia and rigidity were closely associated with the line tracing duration of interval (Table 3; lines 9, 11). $1 \mathrm{~h}$ after L-dopa intake, a correlation appeared between line tracing duration of errors and the tremor score (Table 3; line 22). As to be expected, there were close relationships between the various UPDRS III subscores (results not shown).

\section{Discussion}

Particularly, bradykinesia and rigidity show execution of movement series. These two motor symptoms respond to dopamine substitution quite well. However, both the handwriting procedure and the instrumental test performances do not only depend on velocity. The initiation and conduction of the necessary precise and aimed movement sequences considerably demand cognitive load in the domains' attention and concentration (Lalonde et al. 1997; Espay et al. 2009; Cools et al. 2019). Similar abilities are also needed for the execution of the employed instrumental tasks. Accordingly, we show that all outcomes improved following standardized application of L-dopa/benserazide. We suggest this easy-to-perform handwriting test as an additional tool to supplement the clinical examination of a PD patient when the L-dopa response is tested, i.e., as an essential criterion of the diagnosis of PD (Gelb et al. 1999; Navailles et al. 2014; Trujillo et al. 2019). One must acknowledge that both handwriting and the employed apparatus methods only focus on fine motor behavior of the right hand, which limits their suitability for the clinical use in the case of PD patients with onset of predominant or only left-sided motor symptoms. Prior investigations on handwriting and associated micrographia with digital systems employed tablets with electronic pens. These trials mostly focussed on script height to distinguish PD patients from healthy controls, whereas the speed of task execution sometimes played a minor role in the data analysis (Popovic et al. 2008; Ponsen et al. 2008; Bidet-Ildei et al. 2011; Rosenblum et al. 2013; Cascarano et al. 2019; Zham et al. 2019). The collected data of the handwriting scenario in our present trial mainly assess 
Table 3 Correlation analysis of parameters before and one hour after L-dopa intake and the corresponding computed differences

\begin{tabular}{|c|c|c|c|c|}
\hline Line & Variable 1 & Variable 2 & $R$ & $p$ \\
\hline \multicolumn{5}{|c|}{ Before } \\
\hline 1 & Writing & Peg insertion right & 0,44 & 0,0056 \\
\hline 2 & UPDRS III right arm & Peg insertion right & 0,48 & 0,0021 \\
\hline 3 & UPDRS III right arm & Line tracing errors & 0,37 & 0,0213 \\
\hline 4 & UPDRS III right arm & Line tracing duration of errors & 0,66 & $<0.0001$ \\
\hline 5 & UPDRS III right arm & Line tracing total interval & 0,39 & 0,0143 \\
\hline 6 & Peg insertion right & Line tracing errors & 0,32 & 0,0477 \\
\hline 7 & UPDRS III right arm bradykinesia & Peg insertion right & 0,48 & 0,0024 \\
\hline 8 & UPDRS III right arm bradykinesia & Line tracing duration of errors & 0,62 & $<0.0001$ \\
\hline 9 & UPDRS III right arm bradykinesia & Line tracing total interval & 0,42 & 0,0086 \\
\hline 10 & UPDRS III right arm rigidity & Line tracing errors & 0,33 & 0,0454 \\
\hline 11 & UPDRS III right arm rigidity & Line tracing total interval & 0,52 & 0,0007 \\
\hline 12 & Peg insertion right & Line tracing duration of errors & 0,55 & 0,0004 \\
\hline 13 & Line tracing errors & Line tracing duration of errors & 0,45 & 0,0042 \\
\hline 14 & Line tracing errors & Line tracing total interval & 0,65 & $<0.0001$ \\
\hline 15 & Line tracing duration of errors & Line tracing total interval & 0,58 & 0,0001 \\
\hline \multicolumn{5}{|l|}{ After } \\
\hline 16 & Writing & Line tracing duration of errors & 0,32 & 0,0487 \\
\hline 17 & UPDRS right arm & Peg insertion right & 0,47 & 0,0031 \\
\hline 18 & UPDRS right arm & Line tracing duration of errors & 0,37 & 0,0233 \\
\hline 19 & Peg insertion right & Line tracing duration of errors & 0,60 & $<0.0001$ \\
\hline 20 & Line tracing errors & Line tracing total interval & 0,56 & 0.0003 \\
\hline 21 & UPDRS III right arm bradykinesia & Peg insertion right & 0,51 & 0,0012 \\
\hline 22 & UPDRS III right arm tremor & Line tracing duration of errors & 0,46 & 0,0077 \\
\hline \multicolumn{5}{|c|}{ Difference } \\
\hline 23 & Peg insertion right & Line tracing errors & 0.36 & 0.028 \\
\hline 24 & Line tracing errors & Line tracing total interval & 0.49 & 0.0018 \\
\hline 25 & Line tracing errors & Line tracing duration of errors & 0.37 & 0.024 \\
\hline 26 & Line tracing duration of errors & Line tracing total interval & 0.54 & 0.0005 \\
\hline
\end{tabular}

after $1 \mathrm{~h}$ after application of soluble $100 \mathrm{mg} \mathrm{L}$-dopa/25 mg benserazide, before before intake of soluble $100 \mathrm{mg} \mathrm{L}$-dopa/25 mg benserazide, difference computed differences between the outcomes of the two assessment moments according to the formula (outcome before - outcome after $=$ difference); $R$ correlation coefficient, $p p$ value, UPDRS arm partial arm score motor examination (III) of the Unified Parkinson's Disease Rating Scale writing velocity only. We stress that handwriting also asks for readability in the specific investigational situation, which is considerably influenced by precise trajectories with their considerable impact on size of letters, speed, and fluency of writing (Nackaerts et al. 2017a, b). Coordinated performance and accurated performance of motion sequences are characteristics, which are also important for the execution of the peg insertion test. This task demands to insert a peg into a hole with an aimed movement pattern (Müller et al. 2002). The instrumental determination of number of errors and duration of errors in the line tracing task in particular mirrors the functional capacity for execution of courses of aimed and thus precise movement series (Müller et al. 2000; Nieoullon et al. 2003). Accordingly, peg insertion outcomes showed close correlations to the duration of errors of the line tracing task mainly. Unlike the handwriting task, both peg insertion and line tracing are performed from the right to the left side. Inserting of pegs with its repeat performance and hand writing are influenced by training, which is different from the single carrying out of the line tracing task (Lalonde et al. 1997). This effect may hypothetically explain the missing association between handwriting and line tracing. Both instrumental test results were closely related to the UPDRS part III rating scores of motor behavior, which mainly reflect tremor, bradykinesia, and rigidity. One may assume that they also reflect the speed and precision of movement execution in a more indirect fashion. The aforementioned associations appeared before L-dopa intake. Following L-dopa intake, correlations coefficients of these associations were lower. The computed differences only showed relevant significant correlations within the various parameters of the line tracing task. Reasons for these missing relations may be that 
we assessed the L-dopa response one time only. We did not consider the different onset and time to maximum response to L-dopa. This effect is influenced by various components, such as duodenal absorption, gastrointestinal motility, and amino acid transport system activity. Repeated evaluations, i.e., every $30 \mathrm{~min}$ up to $2 \mathrm{~h}$ after L-dopa intake in view of the L-dopa plasma half-life, would have improved the quality of the present investigation (Müller et al. 2003). A further limitation is that we did not perform this trial in healthy controls to demonstrate or to exclude the learning effects and in previously untreated PD patients. As we included only treated PD patients, we cannot definitely exclude an effect of a long-duration response of the concomitantly performed PD drug therapy despite the $12 \mathrm{~h}$ lasting washout period. Therefore, we suggest further research in previously untreated PD patients. These future investigations will aim to confirm whether line tracing duration of error outcomes may serve as a specific marker for tremor and bradykinesia. Such a study will also focus on further putative relationships between scores of rigidity, respectively, bradykinesia and the various line tracing parameters.

In conclusion, we show that the performance of a simple handwriting paradigm in combination with resembling instrumental tasks may reflect an improvement of the velocity of movement execution following the administration of soluble L-dopa/benserazide in previously treated PD patients.

Acknowledgements We thank Tanja Steiner, Bettina Marchewitz, Gudrun Edler, Ute Claussnitzer, and Christine Stamm for technical assistance. We thank the participating PD patients.

Funding Open Access funding provided by Projekt DEAL.

Open Access This article is licensed under a Creative Commons Attribution 4.0 International License, which permits use, sharing, adaptation, distribution and reproduction in any medium or format, as long as you give appropriate credit to the original author(s) and the source, provide a link to the Creative Commons licence, and indicate if changes were made. The images or other third party material in this article are included in the article's Creative Commons licence, unless indicated otherwise in a credit line to the material. If material is not included in the article's Creative Commons licence and your intended use is not permitted by statutory regulation or exceeds the permitted use, you will need to obtain permission directly from the copyright holder. To view a copy of this licence, visit http://creativecommons.org/licenses/by/4.0/.

\section{References}

Andrich J, Saft C, Ostholt N, Müller T (2007) Complex movement behaviour and progression of Huntington's disease. Neurosci Lett 416:272-274

Bertoli M, Croce UD, Cereatti A, Mancini M (2019) Objective measures to investigate turning impairments and freezing of gait in people with Parkinson's disease. Gait Posture 74:187-193
Bidet-Ildei C, Pollak P, Kandel S, Fraix V, Orliaguet JP (2011) Handwriting in patients with Parkinson disease: effect of L-dopa and stimulation of the sub-thalamic nucleus on motor anticipation. Hum Mov Sci 30:783-791

Broeder S, Nackaerts E, Nieuwboer A, Smits-Engelsman BC, Swinnen SP, Heremans E (2014) The effects of dual tasking on handwriting in patients with Parkinson's disease. Neuroscience 263:193-202

Cascarano GD, Loconsole C, Brunetti A, Lattarulo A, Buongiorno D, Losavio G, Sciascio ED, Bevilacqua V (2019) Biometric handwriting analysis to support Parkinson's Disease assessment and grading. BMC Med Inform Decis Mak 19:252

Cools R, Frobose M, Aarts E, Hofmans L (2019) Dopamine and the motivation of cognitive control. Handb Clin Neurol 163:123-143

Espay AJ, Beaton DE, Morgante F, Gunraj CA, Lang AE, Chen R (2009) Impairments of speed and amplitude of movement in Parkinson's disease: a pilot study. Mov Disord 24:1001-1008

Fahn S, Elton R, Members of the UPDRS Development Committee (1987) Unified Parkinson's disease rating scale. Macmillan, New York, pp 153-163

Gangadhar G, Joseph D, Srinivasan AV, Subramanian D, Shivakeshavan RG, Shobana N, Chakravarthy VS (2009) A computational model of Parkinsonian handwriting that highlights the role of the indirect pathway in the basal ganglia. Hum Mov Sci 28:602-618

Gelb DJ, Oliver E, Gilman S (1999) Diagnostic criteria for Parkinson disease. Arch Neurol 56:33-39

Goetz CG, Stebbins GT, Wolff D, Deleeuw W, Bronte-Stewart H, Elble R, Hallett M, Nutt J, Ramig L, Sanger T, Wu AD, Kraus PH, Blasucci LM, Shamim EA, Sethi KD, Spielman J, Kubota K, Grove AS, Dishman E, Taylor CB (2008) Testing objective measures of motor impairment in early Parkinson's disease: feasibility study of an at-home testing device. Mov Disord 24:549-554

Haaland KY, Elsinger CL, Mayer AR, Durgerian S, Rao SM (2004) Motor sequence complexity and performing hand produce differential patterns of hemispheric lateralization. J Cognit Neurosci $16: 621-636$

Lalonde R, Botez-Marquard T (1997) The neurobiological basis of movement initiation. Rev Neurosci 8:35-54

Lange KW, Mecklinger L, Walitza S, Becker G, Gerlach M, Naumann $\mathrm{M}$, Tucha $\mathrm{O}$ (2006) Brain dopamine and kinematics of graphomotor functions. Hum Mov Sci 25:492-509

Lee W, Evans A, Williams DR (2016) Validation of a smartphone application measuring motor function in Parkinson's disease. J Parkinsons Dis 6:371-382

Li M, Li P, Tian S, Tang K, Chen X (2018) Estimation of temporal gait parameters using a human body electrostatic sensing-based method. Sensors (Basel) 18

Lopane G, Mellone S, Corzani M, Chiari L, Cortelli P, CalandraBuonaura G, Contin M (2018) Supervised versus unsupervised technology-based levodopa monitoring in Parkinson's disease: an intrasubject comparison. J Neurol 265:1343-1352

Müller T, Schafer S, Kuhn W, Przuntek H (2000) Correlation between tapping and inserting of pegs in Parkinson's disease. Can J Neurol Sci 27:311-315

Müller T, Benz S, Przuntek H (2002) Tapping and peg insertion after levodopa intake in treated and de novo parkinsonian patients. Can J Neurol Sci 29:73-77

Müller T, Benz S, Börnke C, Russ H, Przuntek H (2003) Repeated rating improves value of diagnostic dopaminergic challenge tests in Parkinson's disease. J Neural Transm (Vienna ) 110:603-609

Müller T, Kuhn W, Przuntek H (2005) Efficacy of budipine and placebo in untreated patients with Parkinson's disease. J Neural Transm 112:1015-1023

Müller T, Öhm G, Eilert K, Möhr K, Rotter S, Haas T, Küchler M, Lütge S, Marg M, Rothe H (2017) Benefit on motor and nonmotor behavior in a specialized unit for Parkinson's disease. J Neural Transm (Vienna) 124:715-720 
Nackaerts E, Broeder S, Pereira MP, Swinnen SP, Vandenberghe W, Nieuwboer A, Heremans E (2017a) Handwriting training in Parkinson's disease: a trade-off between size, speed and fluency. PLoS ONE 12:e0190223

Nackaerts E, Heremans E, Smits-Engelsman BC, Broeder S, Vandenberghe W, Bergmans B, Nieuwboer A (2017b) Validity and reliability of a new tool to evaluate handwriting difficulties in Parkinson's disease. PLoS ONE 12:e0173157

Navailles S, Di GG, De DP (2014) Predicting dopaminergic effects of L-DOPA in the treatment for Parkinson's disease. CNS Neurosci Ther 20:699-701

Nieoullon A, Coquerel A (2003) Dopamine: a key regulator to adapt action, emotion, motivation and cognition. Curr Opin Neurol 16(Suppl 2):S3-S9

Pal PK, Lee CS, Samii A, Schulzer M, Stoessl AJ, Mak EK, Wudel J, Dobko T, Tsui JK (2001) Alternating two finger tapping with contralateral activation is an objective measure of clinical severity in Parkinson's disease and correlates with PET. Parkinson Relat Disord 7:305-309

Ponsen MM, Daffertshofer A, Wolters EC, Beek PJ, Berendse HW (2008) Impairment of complex upper limb motor function in de novo Parkinson's disease. Parkinsonism Relat Disord 14:199-204

Popovic MB, Dzoljic E, Kostic V (2008) A method to assess hand motor blocks in Parkinson's disease with digitizing tablet. Tohoku J Exp Med 216:317-324
Ringendahl H (2002) Factor structure, normative data and retest-reliability of a test of fine motor functions in patients with idiopathic Parkinson's disease. J Clin Exp Neuropsychol 24:491-502

Rosenblum S, Samuel M, Zlotnik S, Erikh I, Schlesinger I (2013) Handwriting as an objective tool for Parkinson's disease diagnosis. J Neurol 260:2357-2361

Trujillo P, van Wouwe NC, Lin YC, Stark AJ, Petersen KJ, Kang H, Zald DH, Donahue MJ, Claassen DO (2019) Dopamine effects on frontal cortical blood flow and motor inhibition in Parkinson's disease. Cortex 115:99-111

van Uem JM, Isaacs T, Lewin A, Bresolin E, Salkovic D, Espay AJ, Matthews H, Maetzler W (2016) A viewpoint on wearable technology-enabled measurement of wellbeing and health-related quality of life in Parkinson's disease. J Parkinsons Dis 6:279-287

Zham P, Kumar D, Viswanthan R, Wong K, Nagao KJ, Arjunan SP, Raghav S, Kempster P (2019) Effect of levodopa on handwriting tasks of different complexity in Parkinson's disease: a kinematic study. J Neurol 266:1376-1382

Publisher's Note Springer Nature remains neutral with regard to jurisdictional claims in published maps and institutional affiliations. 